\title{
ASPECTOS FISIOLÓgICOS DEL DURAZNERO (Prunus persica [L.] BATSCH) EN EL TRÓPICO ALTO. UNA REVISIÓN
}

\section{PHYSIOLOGICAL ASPECTS OF PEACH (Prunus persica [L.] BATSCH) IN THE HIGH TROPICAL ZONE. A REVIEW}

\author{
Elberth Hernando Pinzón ${ }^{1}$, Ana Cruz Morillo ${ }^{2}$, Gerhard Fischer ${ }^{3}$
}

${ }^{1}$ Ing. Agr., Programa de Maestría en Fisiología Vegetal, Grupo de Investigaciones Agrícolas, Universidad Pedagógica y Tecnológica de Colombia -UPTC-. Av. Central del Norte, Tunja-Colombia, e-mail: elberth02@gmail.com; ${ }^{2}$ Ing. Agr. Ph.D., Facultad de Ciencias Agropecuarias, Universidad Pedagógica y Tecnológica de Colombia -UPTC-. Av. Central del Norte, Tunja-Colombia, e-mail: ana.morillo@uptc.edu.co; ${ }^{3}$ Ing. Hort. Ph.D., Profesor Asociado, Facultad de Ciencias Agrarias, Universidad Nacional de Colombia, Bogotá-Colombia, e-mail: gfischer@unal.edu.co

Rev. U.D.C.A. Act. \& Div. Cient. 17(2): 401-411, Julio-Diciembre, 2014

\section{RESUMEN}

Dentro de las especies caducifolias, el duraznero (Prunus persica [L.] Batsch), proveniente del cercano oriente e introducido por los conquistadores en el siglo XV a Colombia, es uno de los frutales de gran importancia en las zonas de trópico alto. En las áreas altas de estas regiones, las variedades cultivadas han venido sufriendo un cambio en su expresión genética, lo que ha favorecido su adaptación y la implementación de sistemas de producción continuos o de ciclaje. En Colombia, se cultiva en zonas con temperaturas promedio de 13 a $19^{\circ} \mathrm{C}$, entre los 1.800 y $2.800 \mathrm{msnm}$, regímenes de lluvia monomodales y bimodales y brillo solar promedio de 1.400 horas/año. Las variedades más cultivadas son 'Dorado' y 'Diamante', por su bajo requerimiento de horas frío, mientras que variedades como 'Rubidoux' o 'Gran Jarillo', hasta ahora están incursionado, debido a su alto requerimiento de horas frío. El artículo pretende contextualizar la importancia, los cambios fenológicos y ecofisiológicos y los recursos genéticos de una especie tan importante para la fruticultura, en las zonas de trópico alto colombiano.

Palabras clave: Ecofisiología, dormancia, inducción floral, fenología, variedades.

\section{SUMMARY}

Among deciduous tree species peach (Prunus persica (L.) Batsch), from the Near East and introduced by the conquerors in the $15^{\text {th }}$ century to Colombia, is one of the most important fruits in high tropical areas. In highland zones of these regions cultivated varieties have been undergoing a change in gene expression, which has favored the adaptation and implementation of continuous production or cycling systems. In Colombia peach is grown in areas with average temperatures of 13 and $19^{\circ} \mathrm{C}$, located between 1.800 and 2.800 m.a.s.l. with monomodal and bimodal rainfall schemes and an average of 1.400 sunshine hours per year. The most cultivated varieties are 'Gold ' and 'Diamante' because of their low chilling hour requirements, while varieties like 'Rubidoux ' or " Great Jarillo ' are introduced due to its high requirement of chilling hours. The following article seeks to contextualize the importance of phonological and ecophysiological patterns and genetic resources for this important fruit species for the horticulture of the Colombian high altitudinal zones.

Key words: Ecophysiology, dormancy, floral induction, phenology, varieties.

\section{INTRODUCCIÓN}

El duraznero es una de las especies frutales caducifolias más populares que se cultivan en las zonas templadas de todo el mundo. Su nombre científico, Prunus persica (L.) Batsch, sugiere que sería originario de Persia lo que, actualmente, se conoce como Irán (Cárdenas \& Fischer, 2013), pero ya en la literatura China del 2000 A.C., se hacían descripciones de sus flores y frutos maduros, por lo cual, hoy es aceptado, que su origen está en dicho país (Gratacós, 2008). Probablemente, fue llevado de China a Persia por caravanas de comerciantes y luego pasó, rápidamente, a Europa. En el siglo $\mathrm{XVI}$, ya se encontraba en México, traído por los españoles (Gratacós, 2008). 
El duraznero pertenece a la subclase Rosidea, orden Rosales, familia Rosaceae, subfamilia Prunoideae, subgénero Amygdalus (L.) Focke. (USDA, 2013; Cárdenas \& Fischer, 2013). Es uno de los frutales comúnmente sembrados en zonas de trópico alto, dentro de la llamada fruticultura de clima frío, debido a las ventajas comparativas que estas regiones poseen, como clima, suelos, precipitación, acumulación de horas frío, vocación frutícola de los productores y experiencia en el manejo de esta especie (Puentes et al. 2008), que ha permitido su posicionamiento en muchos de los municipios del departamento de Boyacá. Puentes et al. (2008) encuentran indicadores que lo ubican como una alternativa atractiva de inversión para la región.

La producción de duraznero en las zonas de trópico alto colombiano, según la FAOSTAT, para el 2012, fue de 20,174t, con un rendimiento de $13,2 \mathrm{t} \mathrm{ha}^{-1} \mathrm{y}$ un área de siembra de 1.520ha. El departamento de Boyacá sobresale como productor por excelencia de frutales caducifolios, entre los que se destacan ciruelo, peral, manzano, vid y duraznero, siendo este último, una de las especies mejor adaptadas a las condiciones tropicales. Se destacan, especialmente, las zonas altas tropicales, en las que los durazneros pueden encontrar condiciones agroclimáticas adecuadas para su desarrollo (George \& Erez, 2000); sumado a esto, Boyacá es el principal productor, con una participación del $60,5 \%$ del total de la producción nacional, que se distribuye en los municipios de Sotaquirá, Paipa, Tinjacá, Nuevo Colon y Tuta (MADR, 2012); sin embargo, desde la entrada al país, en 1985, de algunas variedades de duraznero procedentes, especialmente de Brasil, entre las que se cuentan 'Dorado', 'Diamante', 'Rey Negro', 'Rubidoux', gracias al proyecto Colombo-Alemán, estableciendo estos materiales a nivel de parcela demostrativa en la Granja Tunguavita de la Universidad Pedagógica y Tecnológica de Colombia (UPTC) Paipa-Boyacá (Fischer, 1992), la investigación y el desarrollo en esta especie ha sido nula o muy escasa.

En la actualidad, el cultivo tiene grandes retos que están relacionados con: cambio climático, reducción de insumos, ya sea de control fitosanitario o fertilización, tecnologías pre y poscosecha y también introducción de nuevos materiales de siembra, adaptados a condiciones ambientales específica (Fachinello et al. 2011).

Considerando la gran importancia de este cultivo en los sistemas productivos de trópico alto, se realizó esta revisión, con el objetivo de presentar el estado actual y analizar los principales aspectos fisiológicos y ecofisiológicos del duraznero, en los sistemas de producción, en las zonas de trópico alto colombiano.

\section{MATERIALES Y MÉTODOS}

La revisión, se basó en el análisis de la información existente sobre el comportamiento fisiológico del duraznero, resultado de investigaciones publicadas de artículos científicos, libros y documentos sobre las especies caducifolias en las zonas tropicales y subtropicales americanas, durante los últimos 15 años, teniendo como eje central la especie $P$. persica, debido a la gran importancia que ha adquirido como opción de desarrollo frutícola, en las zonas del trópico alto colombiano.

El documento le da gran relevancia a los trabajos realizados por investigadores nacionales e internacionales, que con su producción científica han aportado al entendimiento de algunos de los fenómenos fisiológicos que presenta el duraznero, en condiciones de zona ecuatorial o tropical.

\section{RESULTADOS Y DISCUSIÓN}

Producción de duraznero en trópico alto colombiano: Desde la llegada del duraznero a Colombia, por el intercambio de especies vegetales entre Europa y América, llevada a cabo por los conquistadores, entre el siglo XV y XVI, fue cultivado en los valles de Tundama y Sugamuxi, ubicados en el departamento de Boyacá, debido a que estas zonas presentan condiciones similares a las de procedencia, situación que permitió que la especie $P$. persica fuera establecida en estas regiones $\mathrm{y}$, con el tiempo, acogida por los productores como cultivo y luego extenderse a otras zonas del país (Patiño \& Miranda, 2013).

La producción de $P$. persica en Colombia, se concentra en los departamentos de Boyacá, Cundinamarca, Norte de Santander y Santander (MADR, 2012), en municipios ubicados entre 1.600 y 3.332 msnm (Carranza \& Miranda, 2013). El departamento de Boyacá es el mayor productor de duraznero, con una producción de $12.00 \mathrm{t}$, un rendimiento de $14,4 \mathrm{t}$ ha $^{-1}$, en un área de siembra de 833ha (Tabla 1), lo que representa una participación dentro del mercado nacional del $60,5 \%$, que se distribuye en cuatro municipios, siendo Sotaquirá el mayor productor, a nivel nacional, ya que participa con el $47 \%$, seguido de Paipa, con un 13,3\%, Tinjacá, con un 12,1\% y Nuevo Colón, con el 10,1\% (MADR, 2012).

Norte de Santander se ha venido posicionando como un departamento con una gran vocación hacia los frutales caducifolios (Tabla 1), destacándose la incorporación de nuevas áreas de siembra de duraznero, tendencia que se evidencia, ya que se pasó de $168 \mathrm{ha}$, reportadas en 2007 , a $463 \mathrm{ha}$, para el 2011, mostrando un incremento del $285 \%$ en el área y una clara preferencia a seguir incorporando zonas a la producción de esta especie; su participación en el mercado nacional es del 27,2\% (MADR, 2012). 
Tabla 1. Área, producción, rendimiento y variedades cultivadas de duraznero en Colombia (MADR, 2012; Miranda \& Carranza, 2013).

\begin{tabular}{|c|c|c|c|l|}
\hline Departamento & Área (ha) & Producción (t) & $\begin{array}{c}\text { Rendimiento } \\
\text { (t ha-1) }\end{array}$ & Variedades cultivadas \\
\hline Boyacá & 833 & 12.009 & 14,4 & $\begin{array}{l}\text { Dorado, Diamante, Rubidoux, Rey } \\
\text { negro }\end{array}$ \\
\hline Cundinamarca & 167 & 2.028 & 12,11 & Dorado y Diamante \\
\hline Norte de Santander & 463 & 5.395 & 11,6 & Jarillo y Gran Jarillo \\
\hline Santander & 20 & 374 & 18,7 & Jarillo y Gran Jarillo \\
\hline
\end{tabular}

Aspectos fenológicos y de manejo del duraznero en el trópico alto: El duraznero proveniente de zonas templadas tiene un ciclo fenológico diferente a los cultivos tropicales propiamente dichos, presentando unos estados bien marcados entre cosechas, caída foliar, reposo, inducción floral, hinchamiento de yemas, floración, crecimiento y desarrollo y cosecha (Castro \& Puentes, 2012).

Caída foliar y defoliación: La defoliación es un proceso determinante en la programación de la cosecha, ya que se logra adelantar o atrasar la misma y se presenta de forma natural en las zonas de origen. Castro \& Puentes (2012) indican que en las zonas de trópico y subtrópico, donde no existen las condiciones de fotoperiodo y temperatura que induzcan la defoliación de esta especie, los árboles no logran defoliarse en un cien por ciento.

Castro \& Puentes (2012) recomiendan inducir este proceso cuando el árbol por sí sólo se ha defoliado, en más de un $60 \%$, de forma manual o química. Mencionan que en las zonas productoras son usados productos, como oxicloruro de cobre, 5,5 $\mathrm{g} \mathrm{L}^{-1}$, en mezcla con sulfato de zinc, de 7,5 a $10 \mathrm{~g}$ $\mathrm{L}^{-1}$ (la cantidad varía según el porcentaje de defoliación natural y el clima) o con clorato de sodio (defoliante inorgánico), de 4 a 8L ha ${ }^{-1}$. Por su parte, Fischer et al. (2010) mencionan combinaciones de dos sulfatos: sulfato de zinc y sulfato de hierro o también Kocide ${ }^{\circledR} 101$.

Desde el punto de vista fisiológico, la abscisión foliar tiene el mayor efecto sobre la brotación cuando las plantas se manejan bajo el esquema de cosecha continua, en donde, a través de la defoliación, se eliminan inhibidores presentes en las hojas, como el ácido abscísico, aumentando la actividad de promotores, como las giberelinas y citoquininas. De esta manera, se promueve la brotación de las yemas florales (Edwards, 1985); sin embargo, los deshojes demasiado tempranos pueden inhibir la iniciación floral, además de producir flores anormales (Lloyd \& Couvillon, 1974) y su posterior abscisión, razón, por la cual, no es recomendable defoliar los árboles antes de un mes, luego de la cosecha (Castro \& Puentes, 2012).
Dormancia y acumulación de horas frío: La dormancia, se puede definir como la suspensión temporal del crecimiento visible en cualquier estructura de la planta que contiene un meristemo (Lang et al. 1987). El duraznero presenta una serie de mecanismos que le permiten soportar los cambios climáticos, en las zonas templadas. Estos inducen respuestas fisiológicas, entre las que se encuentran la dormancia y el balance entre inhibidores y promotores del crecimiento ( $\mathrm{Ra}$ mírez \& Kallarackal, 2014). La posibilidad de formar yemas y experimentar ciclos de crecimiento y dormancia ha sido una adaptación evolutiva de suma importancia, en el proceso de supervivencia (Rohde \& Bhalerao, 2007).

Por tanto, en las zonas de trópico alto en donde se practica el sistema de cosecha continua o ciclaje se da una brotación constante, ya que las yemas florales se diferencian al poco tiempo, luego de la recolección y están listas para brotar; esto se presenta, debido a que la planta se encuentra en predormancia, cuando las yemas aún no han acumulado tantas sustancias inhibidoras (Sherman \& Lyrene, 1984). Es así, como se puede inducir, de manera forzada, un nuevo ciclo de crecimiento, mediante la eliminación de fuentes de inhibición, antes de que las plantas, eventualmente, entren en endodormancia (Westwood, 1993); no obstante, la expresión génica de los procesos de dormancia en caducifolios se pueden dar en diferentes épocas del año, como una medida de supervivencia (Rom, 2003).

La acumulación de horas frío posibilita los cambios fisiológicos responsables de la floración, la foliación y la fructificación normal en caducifolios (Gil-Albert, 1992; Melgarejo et al. 1997). Esta acumulación varía de acuerdo a la especie o variedad y, se estima, contando el número de horas necesarias para salir del periodo de reposo o dormancia a temperaturas inferiores a un umbral, comprendido entre 4 y $12^{\circ} \mathrm{C}$. Campoy et al. (2011) sugieren que el umbral de $7^{\circ} \mathrm{C}$, es un parámetro apropiado para este tipo de frutales.

En las zonas tropicales, el sistema de medición de horas frío (HF) no es muy relevante, debido al modelo de producción 
de cosecha continua y a que las plantas no entran en endodormancia (Westwood, 1993). Una vez las plantas se hallan en este estado, se hace necesario acumular cierto número de horas frío o unidades de frío, dependiendo del modelo de cálculo, para salir de este estadio de reposo o dormancia (Real, 1987).

En los trópicos, se adapta mejor el modelo Utah, de Richardson et al. (1974), porque incluye el efecto de horas que pasan los $7,2^{\circ} \mathrm{C}$ y no tiene en cuenta las horas frío recibidas en la noche, cuando las horas calor son demasiado altas en el día. Las unidades de frío (UF), se definen como el aporte que realiza una hora transcurrida en el intervalo térmico, de 2,5 a $9,1^{\circ} \mathrm{C}$, para que termine la dormancia de la planta (Gariglio et al. 2006). Según Gariglio et al. (2007), para duraznero, el requerimiento de HF está entre 100 a 1100, dependiendo de la variedad; las más cultivadas, se hallan entre 600 y 800 HF; sin embargo, Chaar \& Astorga (2012) encontraron en algunas variedades de duraznero una amplia variabilidad interanual en los requerimientos de frío y de calor de un mismo cultivar en un mismo sitio, lo que pone en evidencia la importancia de evaluar los materiales vegetales con respecto a la relación entre sus requerimientos térmicos, ya que, debido al cambio climático no son constantes o bien promediar los valores obtenidos, de una serie extensa de años.

Inducción y diferenciación floral: En duraznero, la inducción floral ocurre, normalmente, muy cerca de la cosecha, en ramas del último crecimiento, que aún no han producido frutos. Leite et al. (2004) sugieren que para entender el patrón de brotación de yemas florales en el duraznero, se deben tener en cuenta factores, como la capacidad para usar los azúcares solubles, para lo cual, se podría tomar, como indicador, la capacidad de síntesis de almidón almacenado en el leño. Al respecto, Fischer et al. (2010) indican que cuando el árbol se encuentra en estados fenológicos desfasados y traslapados, como sucede por falta de frío o después de una sequía, las yemas pueden abrirse antes de la cosecha y dificultan el manejo adecuado, en sistemas de cosechas continuas.

Durante la diferenciación, las yemas generativas tienen que pasar una serie de estados que, por lo general, están completamente terminados cuando se hinchan; esto ocurre poco antes de la brotación. Se debe tener en cuenta que en los caducifolios, una yema plenamente diferenciada durante el ciclo vegetativo no va a brotar, mientras existan hojas en pleno crecimiento, que produzcan hormonas inhibidoras, como el ácido abscísico (Fulford, 1970).

En los caducifolios es importante evitar una sobrecarga de frutos para garantizar una adecuada formación de las yemas florales para el siguiente ciclo (Fischer, 2013). En zonas productoras de trópico alto, la relación hoja/fruto es un factor determinante de la calidad del fruto de duraznero. Casierra et al. (2007) encontraron que en la variedad 'Rubidoux', la relación está entre 40 a 50 hojas por fruto, siendo adecuada para obtener buenos resultados, en cuanto a características fisicoquímicas, como sólidos solubles y relación pulpa/semilla, constituyendo un buen criterio para el raleo de frutos, en esta variedad.

El duraznero requiere de condiciones térmicas específicas para el proceso de antesis y temperaturas nocturnas moderadas, que no deben superar los $14^{\circ} \mathrm{C}$ (George et al. 1988). En zonas de trópico bajo, debido al régimen de temperaturas diurnas superiores a $25^{\circ} \mathrm{C}$ o temperaturas nocturnas $>18^{\circ} \mathrm{C}$, se presenta un cuajamiento deficiente de frutos (Edwards, 1987; Erez et al. 1993). En P. persica como en otras drupáceas, la temperatura promedio del mes más frío no debe ser mayor de $13^{\circ} \mathrm{C}$ ni inferior a $1^{\circ} \mathrm{C}$, debido a que se causaría daño o restricción del desarrollo de los frutos (George et al. 1988; Erez et al. 1993; Ramírez \& Kallarackal, 2014).

Dennis (2000) resume varias condiciones tendientes a garantizar una buena floración en zonas de trópico y subtrópico: evitar temperaturas altas (excepción en la vid), garantizar una intensidad lumínica suficiente (fotoperiodo), ralear frutos en árboles altamente cargados, reducir suministro de agua (para reducir el crecimiento vegetativo) y agobio de ramas (especialmente, en manzanos y en perales).

Crecimiento y desarrollo: En duraznero, el crecimiento del pericarpio sigue un patrón doble sigmoide, con dos periodos de incremento rápido y una fase intermedia de crecimiento, aparentemente suspendido (Casierra et al. 2004). El crecimiento de los frutos de durazno, tradicionalmente, se ha dividido en tres etapas: la primera, está marcada por el incremento de la mitosis en el fruto, durante las dos o tres primeras semanas de su desarrollo y, luego, se reduce drásticamente (Grange, 1996); la segunda etapa, se caracteriza por un incremento escaso en el mesocarpio, reducción general del crecimiento, cesa la elongación celular, tanto en el plano tangencial como en el longitudinal (Coombe, 1976); el evento más importante en esta etapa es la lignificación del endocarpio, que comienza a finales de la primera etapa de crecimiento del fruto y se acentúa en la segunda y, en algunos casos, según la variedad, se prolonga hasta la tercera etapa. En la etapa tres, la última fase de crecimiento de los frutos, se muestra un incremento rápido de los pesos seco y fresco en el mesocarpio, la elongación celular continúa, los espacios intercelulares se reducen hasta casi desaparecer al momento de la maduración (Gage \& Stutte, 1991).

En las variedades tardías, el endocarpio alcanza su máximo peso durante la primera parte de esta etapa y la ganancia en peso seco de la semilla es muy rápida y dura hasta la maduración. El contenido de sólidos solubles, que se había redu- 
cido a raíz de la elongación celular, se incrementa de nuevo (Chalmers \& Van den Ende, 1975). Se reportan tiempos de desarrollo del fruto de 139 y 153 días, respectivamente, para las variedades 'Diamante' y ‘Dorado' (Russel \& Topp, 2002).

Aspectos ecofisiológicos: La ecofisiología del duraznero está ligada a su centro de origen, el cual, se ubica en el cercano oriente, lo que comprendía la antigua Persia (Harlan, 1971; Cárdenas \& Fischer, 2013), en latitudes de $30^{\circ}$ a $50^{\circ}$, que corresponden a zonas templadas, donde registra las condiciones edafoclimáticas adecuadas, para su normal crecimiento y desarrollo; sin embargo, en las zonas tropicales se ve limitado, ya que la temperatura y el fotoperiodo son relativamente uniformes durante todo el año, lo que no hace parte del normal desarrollo fisiológico de esta especie, que requiere de cambios bruscos en las condiciones climáticas, para realizar su ciclo natural (Westwood, 1993). Por tanto, la producción de esta especie en el trópico, se afecta de forma considerable (Erez, 2000).

Temperatura y altitud: La temperatura es el factor que quizás más afecta la producción de $P$. persica, ya que en la zona templada la producción se maneja bajo la técnica de única cosecha, en la que el árbol entra en una dormancia profunda y requiere de temperaturas entre 6 y $8^{\circ} \mathrm{C}$, para satisfacer los requerimiento de $\mathrm{HF}$, lo que coincide con la estación de invierno (Erez \& Lavee, 1971). En duraznero, el tener temperaturas que no estén por debajo de $10^{\circ} \mathrm{C}$ es de vital importancia para que las raíces no disminuyan su actividad, de esta manera, se evita que las plantas entren en procesos de endodormancia (Erez, 1986).

Se reporta que para el crecimiento del cultivo en sistemas comerciales se necesitan temperaturas anuales mínimas de 12 a $13^{\circ} \mathrm{C}$ (Fischer, 1992). Temperaturas promedio entre 14 y $20^{\circ} \mathrm{C}$ han favorecido la producción continua de caducifolios en Colombia, en donde se ha encontrado que temperaturas alrededor de $18^{\circ} \mathrm{C}$ son adecuadas para el crecimiento, la floración y el desarrollo del fruto en caducifolios (Fischer, 1992). En las zonas productoras de Colombia, puntualmente en el departamento de Boyacá, se presentan temperaturas medias mínimas de 6,2 a $8,4^{\circ} \mathrm{C}$ y medias máximas de 23,4 a $24,9^{\circ} \mathrm{C}$ (Fischer, 1993a).

El sistema de producción en las zonas tropicales es de cosecha forzada o continua, lo que implica que la temperatura debe ser uniforme durante todo el año y no se debe presentar estación fría, eventualmente, causada por una época lluviosa prolongada, como sucede en algunas zonas, debido al régimen monomodal de precipitaciones (Fischer, 2013).

La diferenciación floral se puede ver afectada en zonas con presencia de altas temperaturas, ya que esto favorece la producción de giberelinas en los puntos de crecimiento foliares
(Tromp, 1976). Por su parte, Edwards (1987) y Erez et al. (1993) registraron que en zonas tropicales con temperaturas diurnas superiores a $25^{\circ} \mathrm{C}$ o temperaturas nocturnas por encima de $18^{\circ} \mathrm{C}$, el proceso de antesis se ve afectado en esta especie, ocasionando un reducido cuajamiento de frutos. Las cosechas continuas o ciclaje en el trópico son posibles, porque en estas latitudes se ubican en la planta hojas fotosintéticamente activas durante 11 meses, mientras en los subtrópicos, este tiempo es de 8 a 9 meses y, en las zonas templadas, es de 6 a 7 meses (George \& Erez, 2000).

El duraznero alcanza su potencial de rendimiento en el trópico cuando se presenta una larga temporada de crecimiento, estimulada por temperaturas moderadas en el día y combinadas con temperaturas nocturnas baja, ya que cuando estas últimas son altas, la respiración de mantenimiento aumenta, elevando el costo energético de la planta y disminuyendo el balance neto de carbono día (Gariglio et al. 2007).

La altitud es un factor que tiene una relación directa sobre la temperatura; Boshell (1982) reporta que la temperatura disminuye con el aumento de la altitud, unos $0,6^{\circ} \mathrm{C}$, por cada $100 \mathrm{~m}$. En especies, como P. pérsica, se observa claramente que cada variedad necesita una elevación mínima para lograr satisfacer sus necesidades de horas frío (Westwood, 1993).

El cultivo comercial de duraznero en Colombia, se ubica en zonas que se encuentran en altitudes entre 1.600 y 3.332msnm (Carranza \& Miranda, 2013); no obstante, en sitios entre 2.300 y $2.600 \mathrm{msnm}$, como en los departamentos de Boyacá y Cundinamarca, se presenta el fenómeno conocido como heladas, en el que se presentan temperaturas por debajo de $0^{\circ}$, fenómeno climático que debe resolver el fruticultor, ya que afectaría la floración y el cuajamiento de los frutos (Fischer, 2013).

Relaciones hídricas: Del Ángel et al. (2001) consideran que el crecimiento y la productividad de los cultivos está en función del uso eficiente del recurso hídrico, ya que un déficit marcado en el suelo afecta la producción final; aunque en algunas especies sometidas a un estrés hídrico moderado puede llevar a mejorar la eficiencia del uso del agua y la calidad de los productos.

Teniendo en cuenta que en las zonas de trópico alto el sistema de producción es de cosecha continua y que en la mayoría de las regiones productoras el régimen de lluvias es bimodal, es decir, las lluvias están repartidas en dos épocas del año, Fischer (1993b) indica que estas condiciones son las más favorables, puesto que se tiene una periodo seco que favorece la fase de posrecolección y, épocas de lluvia, que son esenciales en la fase de desarrollo y de crecimiento del fruto. En las zonas productoras de Boyacá, la precipitación pluvial anual oscila entre 700 y 1400mm al año que, según George 
\& Erez (2000), son las condiciones indicadas para mantener los árboles sanos y buena calidad de los frutos, dado que las zonas más apropiadas son aquellas que reciben menos de $1.500 \mathrm{~mm}$ de lluvia, en la fase reproductiva del árbol.

Aunque se cuenta con las condiciones adecuadas, en muchas de las zonas productoras, debido al cambio climático, los regímenes de lluvia han cambiado, teniendo años con déficit seguidos por años con excesos e inundaciones; debido a esto, los agricultores han venido implementando sistemas de riego, con el fin de dar un mejor manejo del agua y así garantizar la cosecha. Según Puppo \& García (2010), un manejo correcto del riego permite apuntar a altos rendimientos, con estabilidad entre años y una calidad óptima del producto cosechado, haciendo posible un uso racional del agua y de la energía, de esta forma, reducir el desperdicio de estos recursos y la contaminación del medio ambiente.

Morales et al. (2010) apreciaron que la aplicación del riego mediante el sistema de goteo contribuye significativamente al aumento del tamaño de la fruta y afecta parámetros fisiológicos, como potencial hídrico foliar y xilemático y conductancia estomática. Bryla et al. (2005) indican que en duraznero, el sistema de riego por goteo ha mostrado los mejores resultados, ya que proporciona una humedad más uniforme que los sistemas de riego por gravedad y por aspersión.

En contraste, los excesos de agua pueden ocasionar daños a nivel radicular, debido a que las raíces del duraznero son muy sensibles a la anoxia, ya que exhiben un tipo de enraizamiento superficial (Reighard et al. 2001).

González et al. (2006) encontraron que condiciones de anegamiento afectan el metabolismo y el cuajado de los frutos, puesto que afecta en forma directa parámetros de calidad, como sólidos solubles totales, firmeza y color, además de que los frutos al presentarse esta condición resultaron de menor peso, tanto seco como fresco y se presenta una mayor cantidad de frutos atacados por patógenos, en su mayoría hongos. Fischer et al. (2010) indican que en zonas con altas precipitaciones, se debe evitar que la antesis y el cuajamiento coincidan con un periodo de lluvias muy intenso, debido a que se produciría una floración reducida, así como flores y frutos afectados.

Radiación solar: De los factores climáticos, el brillo solar tiene una íntima relación con la fisiología de la planta, ya que el acortamiento de las horas luz por efectos, como nubosidad o neblina, pueden ocasionar suspensión del crecimiento del árbol o procesos de dormancia profunda.

Fischer (1992) reporta que al presentarse una radiación demasiado alta durante el reposo puede impedir la brotación, debido al aumento de la temperatura de las yemas. De igual forma, Erez (2000) indica que, debido al cambio climático, la radiación solar se ha modificado, ocasionando alteraciones durante el ciclo de crecimiento del árbol y del fruto, que puede afectar su cuajamiento, su rendimiento y su calidad, además de la fotosíntesis y el crecimiento regular de la planta. En las zonas de trópico alto, se ha observado que la luminosidad juega un papel importante en el fenómeno de acumulación o desacumulación de las horas frío. La alta nubosidad y la baja luminosidad en clima tropical alto, impide que las temperaturas diurnas se eleven demasiado, manteniendo así la acumulación de las horas frío de la noche (Fischer, 2013).

Ávila et al. (2013) mencionan que el brillo solar es uno de los factores más importantes en los procesos de adaptación de los caducifolios en zonas de trópico, debido a que este factor induce el reposo en estas especies; indican que en las zonas productoras de Colombia, las horas de brillo solar se encuentran entre 800 y 2.000 , con un promedio anual de $1.400 \mathrm{~h}$; el descenso o incremento en las curvas de brillo solar, coinciden claramente con las épocas fisiológicas más importantes de estos frutales, como son reposo y brotación, que dará como resultado la floración y la foliación del árbol, es decir, que el fotoperiodo en el trópico regula, tanto los estadios de crecimiento vegetativo como reproductivos.

Se puede concluir que $P$. persica al ser un cultivo perenne requiere, para su normal crecimiento, unas condiciones de suelo y de ambientes que no le generen limitaciones, ya que toda alteración afectará el normal desarrollo del sistema radicular, que se evidenciará en la parte aérea de la planta, causando la disminución del crecimiento de la planta y la reducción en la producción, tanto en la cantidad como en la calidad de los frutos (Romo \& Díaz, 1985).

Recursos genéticos: En cuanto a recursos genéticos, para el caso de duraznero en las zonas tropicales de América, estos provienen, en su mayoría, de árboles francos, haciendo referencia a su procedencia, a partir de semillas, traídas por los españoles durante el proceso de conquista y debido a procesos de selección natural o asistida, se han adaptado a las condiciones agroecológicas tropicales (Westwood, 1993).

En Colombia, se cuenta con un banco de germoplasma de frutales caducifolios, creado en 1985, por el convenio Colombo-Alemán, que se estableció en la Granja Tunguavita de la Universidad Pedagógica y Tecnológica de Colombia -UPTC-, en el municipio de Paipa, Boyacá, con el fin de estudiar el comportamiento y la adaptación de diferentes especies y variedades importadas, especialmente de Brasil y de Alemania (Patiño y Miranda, 2013). Dentro de las variedades de duraznero introducidas estaban 'Dorado', 'Diamante', 'Rey Negro', 'Rubidoux', 'Riograndense' y 'Floridas', las cuales, se dispusieron a nivel de parcela demostrativa (Fischer, 1992). 
Dentro del cultivo de duraznero existen variedades tempranas, como 'Diamante' y 'Dorado'; tardías, como 'Rubidoux' y 'Gran Jarillo' e intermedias, como 'Rey Negro' (Tabla 2), que con excelente manejo agronómico y adecuada oferta agroecológica, se pueden ofrecer, tanto para consumo en fresco o como agroindustria para todo el año, favorecida por la ubicación geográfica en el trópico y la diversidad de microclimas presentes en el país (Castro \& Puentes, 2012).
Según Campos (2013), en Colombia, se cuenta con duraznos propiamente dichos, siendo aquellos que tienen la piel aterciopelada y la pulpa está unida al hueso, dentro de los que se encuentran las variedades 'Blanco Común', 'Flor Blanca', 'Floridas', 'Camuezo', 'Conservero Amarillo', 'Riograndense', 'Dorado' y 'Diamante'; Durazno-melocotón, de piel aterciopelada, pero la pulpa no está unida al hueso y se registran las variedades 'Rubidoux', 'Gran Jarillo' y 'Rey

Tabla 2. Características generales de las variedades cultivadas en las zonas de trópico alto colombiano (Campos, 2013).

\begin{tabular}{|l|l|c|l|}
\hline Variedad & $\begin{array}{l}\text { Adaptación } \\
(\mathbf{m s n m})\end{array}$ & $\begin{array}{c}\text { Requerimiento de frío } \\
\left(<7,2 \text { o }<7^{\circ} \mathbf{C}\right)\end{array}$ & Características del fruto \\
\hline Dorado & 2.200 a 2.700 & 300 & $\begin{array}{l}\text { Piel amarilla con ligera pigmentación roja, pulpa amarilla con } \\
\text { coloración rojiza al rededor del hueso. Forma redondeada, peso } \\
\text { promedio de } 150 \mathrm{~g}, \text { consumo en fresco o agroindustria. }\end{array}$ \\
\hline Diamante & 1.800 a 2.700 & 200 & $\begin{array}{l}\text { Piel amarilla con ligera pigmentación rojiza, pulpa amarilla oro, } \\
\text { de forma redondeada; sin embargo, la parte distal termina ligera- } \\
\text { mente puntiaguda. Peso promedio 100g. }\end{array}$ \\
\hline Rubidoux & 2.400 a 2.800 & 500 a 700 & $\begin{array}{l}\text { Piel amarilla con tonalidades rojizas fuertes, pulpa amarilla con } \\
\text { coloración rojiza al rededor del hueso. Forma ligeramente redon- } \\
\text { deada y de tamaño grande, con peso promedio de 150g. Resist- } \\
\text { ente al manipuleo poscosecha. }\end{array}$ \\
\hline Rey negro & 2.200 a 2.700 & 350 a 450 & $\begin{array}{l}\text { Piel amarilla clara con manchas rojas opacas, pulpa blanca con } \\
\text { coloración rojiza alrededor del hueso, de sabor dulce. Forma } \\
\text { ovalada con presencia de pezón muy pronunciado y peso prome- } \\
\text { dio de } 160 \mathrm{~g} .\end{array}$ \\
\hline Gran Jarillo & 2.400 a 2.800 & 500 a 700 & $\begin{array}{l}\text { Piel rojiza con tonalidades amarillas, pulpa amarilla con coloración } \\
\text { rojiza alrededor del hueso. Forma ligeramente redondeada y de } \\
\text { tamaño grande, con peso aproximado de 150g. Presenta buena re- } \\
\text { sistencia a la manipulación poscosecha. Este durazno proveniente } \\
\text { de Venezuela (Colonia Tovar, Jarillo). }\end{array}$ \\
\hline
\end{tabular}

Negro' y Durazno-nectarino, de piel lisa y pulpa no unida al hueso, resultado de una mutación espontánea (Raseira \& Nakasu, 1989). Se indica que a nivel de trópico alto este tipo de durazneros no han tenido una gran aceptación, debido a problemas de adaptación, que ocasionan cuarteamiento de los frutos; sin embargo, se han introducido variedades como 'Arking', 'Rubrosol' y 'Cascata' (Campos, 2013).

Teniendo en cuenta el sistema de producción en las zonas de trópico alto colombiano, las variedades que presentan una mejor expresión génica, en cuanto a adaptación, son aquellas que tienen un requerimiento bajo en horas frío, además que resultan más adecuadas en el manejo de los sistemas de producción continua o de ciclaje (Díaz, 1992); algunas de estas variedades tienen un periodo corto de desarrollo del fruto, como 'Flordaprince', 78 días o 'Flordagold', 88 días, que permite tener ciclos continuos (Fischer, 1993a). Por su parte, variedades como 'Diamante' y 'Dorado', que provienen de Brasil, con un requerimiento de 200 y 300HF, respectivamente, son las más utilizadas por los fruticultores en el departamento de Boyacá; mientras que la variedad 'Rubidoux', con un requerimiento de 600HF (Hawerroth et al. 2009) y un largo desarrollo del fruto, que puede llegar a los 241 días (Casierra et al. 2007) y 'Conservero', que requiere de 196 días, para el desarrollo del fruto, deben ser utilizadas bajo el sistema de única cosecha (Casierra et al. 2004).

Algunos avances en cuanto a nuevas variedades para trópico alto son los reportados por Byrne \& Boonprakob (2008), quienes descubrieron buenas características organolépticas 
en cuatro genotipos de la serie «Thai Tiger TM», con características como frutos de pulpa amarilla, con un sabor agridulce.

Conflictos de intereses: El manuscrito fue preparado y revisado con la participación de los autores, quienes declaramos que no existe conflicto de intereses, que ponga en riesgo la validez de los resultados presentados.

\section{BIBLIOGRAFÍA}

1. ÁVILA, C.A.; ROBLES, A.; PINZÓN, J.; MIRANDA, D.; FISCHER, G. 2013. Tecnologías locales para los sistemas de producción de frutales caducifolios en zonas productoras de Colombia y sus limitantes tecnológicas. En: Miranda, D.; Fischer, G.; Carranza, C. (eds). Los frutales caducifolios en Colombia: Situación actual, sistemas de cultivo y plan de desarrollo. Soc. Col. Cienc. Hort. p.115-150.

2. BYRNE, D.H.; BOONPRAKOB, U. 2008. Thai Tiger TM series of low-chill peaches for the subtropics. HortScience. 43(7):2226-2227.

3. BRYLA, D.R.; DICKSON, E.; SHENCK, R.; JOHNSON, R.S.; CRISOSTO, C.H.; TROUT, T.J. 2005. Influence of irrigation method and scheduling on patterns of soil and tree water status and its relation to yield and fruit quality in peach. HortScience. 40(7):21182124.

4. BOSHELL, J.F. 1982. Condiciones meteorológicas generales de Colombia. En: Fruticultura tropical. Federecafé, Bogotá. p.3-8.

5. CAMPOS, T. DE J. 2013. Especies y variedades de hoja caduca en Colombia. En: Miranda, D.; Fischer, G.; Carranza, C. (eds). Los frutales caducifolios en Colombia: Situación actual, sistemas de cultivo y plan de desarrollo. Soc. Col. Cienc. Hort. p.47-66.

6. CAMPOY, J.; RUIZ, D.; COOK, N.; ALLDERMAN, L.; EGEA, J. 2011. Clinal variation of dormancy progression in apricot. South Afr. J. Bot. 77:618-630.

7. CÁRDENAS, J.; FISCHER, G. 2013. Clasificación botánica y morfología de manzano, peral, duraznero y ciruelo. En: Miranda, D.; Fischer, G.; Carranza, C. (eds). Los frutales caducifolios en Colombia: Situación actual, sistemas de cultivo y plan de desarrollo. Soc. Col. Cienc. Hort. p.24-25.

8. CARRANZA, C.; MIRANDA, D. 2013. Zonificación actual de los sistemas de producción de frutales caducifo- lios en Colombia. En: Miranda, D.; Fischer, G.; Carranza, C. (eds). Los frutales caducifolios en Colombia: Situación actual, sistemas de cultivo y plan de desarrollo. Soc. Col. Cienc. Hort. p.67-86.

9. CASIERRA-POSADA, F.; RODRÍGUEZ, J.I.; CÁRDENAS, J. 2007. La relación hoja: fruto afecta la producción, el crecimiento y la calidad del fruto en duraznero (Prunus persica L. Batsch, cv. 'Rubidoux'). Rev. Fac. Nal. Agr. Medellín 60(1):3657-3669.

10. CASIERRA-POSADA, F.; BARRETO, V.E.; FONSECA, O.L. 2004. Crecimiento de frutos y ramas de duraznero (Prunus persica L. Batsch, cv. 'Conservero') en los altiplanos colombianos. Agro. Col. 22(1):40-45.

11. CASTRO, Á.; PUENTES, G. 2012. Ciruelo y duraznero (Prunus salicina Lindl.) - (Prunus persica (L.) Batsch.). En: Fischer, G. (ed). Manual para el Cultivo de Frutales en el Trópico. Ed. Produmedios, Bogotá. p.370-392.

12. COOMBE, B. 1976. The Development of fleshy fruits. Rev. Plant. Physiol. 27:507-528.

13. CHAAR, J.; ASTORGA, D. 2012. Determinación del requerimiento de frío y de calor en duraznero [Prunus persica (L.) Batsch.] mediante un modelo de correlación. Rev. Investig. Agropec. 38(3):289-298.

14. CHALMERS, D.J.; VAN DEN ENDE, B.A. 1975. Reappraisal of the growth and development of peach fruit. Australian J. Plant Physiol. 2:623-634.

15. DEL ÁNGEL, J.; TIJERINA, L.; ACOSTA, R. 2001. Producción de ciruelo con fertirriego en función de contenidos de humedad y coberturas orgánicas. Terra Latinoam. 19(4):317-326.

16. DENNIS, F.G. 2000. Flowering, fruit set and development under warm conditions. En: Erez, A. (ed). Temperate fruit crops in warm climates. Kluwer Academic Publ., Dordrecht, The Netherlands. p.101-122.

17. DÍAZ, D.H. 1992. Regulación del reposo en duraznero bajo condiciones tropicales y subtropicales. Acta. Hort. 310:83-96.

18. EDWARDS, G.R. 1987. Temperature in relation to peach culture in the tropics. Acta. Hort. 199:61-62.

19. EDWARDS, G.R. 1985. Changes in endogenous hormones in apple during bud burst induced by defoliation. Acta. Hort. 158:203-210. 
20. EREZ, A. 2000. Bud dormancy; phenomenon, problems and solutions in the tropics and subtropics. En: Erez, A. (ed). Temperate fruit crops in warm climates. Kluwer Academic Publ., Dordrecht, The Netherlands. p.17-48.

21. EREZ, A.; YABLEWITZ, Z.; KORCINSKI, R. 1993. High density plantings for protected cultivation of fruit crops; plastic cover for peach and nectarine. Acta Hort. 349:95-98.

22. EREZ, A. 1986. The significance of the length of the leafless stage in deciduous fruit trees on fruit production potential. En: Lakso, A.N.; Lenz, F. (eds). The regulation of photosynthesis in fruit trees. New York State Agricultural Experiment Station, Geneva. p.23.

23. EREZ, A.; LAVEE, S. 1971. The effect of climatic conditions on dormancy development in peach buds. I. Temperatura. J. Amer. Soc. Hort. Sci. 96:711-714.

24. FACHINELLO, J.C.; PASA, M.; SCHMITZ, J.; BETEMPS, D.L. 2011. Situação e perspectivas da fruticultura de clima temperado no Brasil. Rev. Bras. Frutic. 33 ( $\mathrm{N}^{\circ}$ especial):109-120.

25. FISCHER, G. 1992. Present state and development of deciduous fruit culture in Boyacá, Colombia. Acta. Hort. 310:41-49.

26. FISCHER, G. 1993a. Fisiología y manejo en pre y poscosecha. En: Frutales caducifolios manzano, peral, durazno, ciruelo. Ed. Siac-Fenalce, Bogotá. p.97-117.

27. FISCHER, G. 1993b. Fisiología en la producción de dos cosechas anuales en manzano y duraznero. AgroDesarrollo 4(1-2):18-31.

28. FISCHER, G.; CASIERRA-POSADA, F; VILLAMIZAR, C. 2010. Producción forzada de duraznero (Prunus persica (L.) Batsch) en el altiplano tropical de Boyacá (Colombia). Rev. Col. Cienc. Hortíc. 4(1):19-32.

29. FISCHER, G. 2013. Comportamiento de los frutales caducifolios en el trópico. En: Miranda, D.; Fischer, G.; Carranza, C. (eds). Los frutales caducifolios en Colombia: Situación actual, sistemas de cultivo y plan de desarrollo. Soc. Col. Cienc. Hort. p.31-46.

30. FAOSTAT. 2012. Statistic of crop the Peach in Colombia. Disponible desde internet en: http://faostat3.fao.org/ faostat-gateway/go/to/download/Q/QC/S (con acceso $30 / 07 / 2014)$.
31. FULFORD, R.M. 1970. Leaves, fruit and flower initiation. Proc. 18th Int. Hort.l Congress, Tel-Aviv, Israel 4:143150.

32. GAGE, J.; STUTTE, G. 1991. Developmental indices of peach: An anatomical framework. HortScience 26(5), 459- 463.

33. GRANGE, R. 1996. Crecimiento del fruto. En: AzcónBieto, J. y M. Talón (eds.). Fisiología y bioquímica vegetal. Interamericana - McGraw-Hill, Madrid. p.449462.

34. GRATACÓS, E. 2008, El cultivo del duraznero Prunus persica (L.) Batsch. En: Apuntes para la Cátedra de Fruticultura de Hoja Caduca. Pontificia U. Católica Valparaíso. Fac. Agronomía. p.3-4.

35. GARIGLIO, N.; DOVIS, V.; LEVA, P.; GARCÍA, M.; BOUZO, C. 2006. Acumulación de horas de frío en la zona centro oeste centro-oeste de Santa Fe (Argentina) para frutales caducifolios. Hort. Argent. 25(58):2632.

36. GARIGLIO, N.; PILATTI, R.; FONFRÍA, M. 2007. Requerimiento ecofisiológicos de los árboles frutales. En: Sozzi, G.O. (ed). Árboles frutales: Ecofisiología, cultivo y aprovechamiento. Ed. Fac. Agronomía, U. Buenos Aires, Buenos Aires. p.41-82.

37. GEORGE, A.; EREZ, A. 2000. Stone fruit species under warm subtropical and tropical climates. En: Erez, A. (ed). Temperate fruit crops in warm climates. Kluwer Academis Publ., Dordrecht. The Netherlands. p.231265.

38. GEORGE, A.P.; NISSEN, R.; SHERMAN, B. 1988. Overlapping and early single cropping of low chill peaches in Australia. Fruit Var. J. 42(3):91-95.

39. GIL-ALBERT, F. 1992. Tratado de arboricultura frutal. Vol. II. La ecología del árbol frutal. Ediciones MundiPrensa, Madrid. 207p.

40. GONZÁLEZ, M.; MORENO, G.; GIARDINA, E.; DI MIRO, M. 2006. Exceso de agua en el suelo: efecto sobre la calidad del fruto del duraznero Prunus persica (L.) Batsch. Suelo (Argentina) 24(1):1-5.

41. HARLAN, J.R. 1971. Agricultural origins: Centers and non centers. Science (Washington) 174:468-474.

42. HAWERROTH, F.J.; PETRI, J.L.; BERENHAUSER, G.; HERTER, F.G.; MARAFON, A.C. 2009. Efeito do frio 
e do desponte na brotação de gemas em pessegueiro. Rev. Bras. Frutic. 31(2):440-446.

43. LANG, G.A.; EARLY, J.D.; MARTIN, G.C.; DARNELL, R.L. 1987. Endo-, para -,and ecodormancy: physiological terminology and classification for dormancy research. HortScience. 22:371-377.

44. LEITE, G.B.; BONHOMME, M.; LACOINTE, A.; RAGEAU, R.; SAKR, S.; GUILLIOT, A.; MAUREL, K.; PETEL, G.; COUTO-RODRÍGUEZ, A. 2004. Influence of lack of chilling of budbreak pattern and evolution of sugar contents in buds and stem tissues along the oneyear-old shoot of the peach tree. Acta Hort. 662:6171.

45. LLOYD, D.A; COUVILLON, G.A. 1974. Effects of date of defoliation on flower and leaf bud development in the peach Prunus persica (L.) Batsch. J. Amer. Soc. Hort. Sci. 99(6):514-517.

46. MELGAREJO, P.; MARTÍNEZ, V.R.; GUILLAMÓN, J.M.; MIRO, M.; AMORÓS, A. 1997. Phenological stages of the pomegranate tree (Punica granatum L.). Ann. Appl. Biol. 130:135-140.

47. MINISTERIO DE AGRICULTURA Y DESARROLLO RURAL -MADR-. 2012. Anuario estadístico de frutas y hortalizas 2007-2011. Disponible desde internet en: http://www.agronet.gov.co/www/htm3b/public/Anuario/anuario\%20estadistico\%20de\%20frutas\%20y\%20 hortalizas\%202011.pdf (con acceso 20/01/14).

48. MIRANDA, D.; CARRANZA, C. 2013. Caracterización, clasificación y tipificación de los sistemas de producción de caducifolios: ciruelo, duraznero, manzano y peral en zonas productoras de Colombia. En: Miranda, D.; Fischer, G.; Carranza, C. (eds). Los frutales caducifolios en Colombia: Situación actual, sistemas de cultivo y plan de desarrollo. Soc. Col. Cienc. Hort. p.87-114.

49. MORALES, P.; GARCÍA-PETILLO, M.; HAYASHI, R.; PUPPO, L. 2010. Respuesta del duraznero a diferentes patrones de aplicación del agua. Rev. Bras. Eng. Agríc. Ambiental 14(1):17-24.

50. PATIÑO, L.; MIRANDA, D. 2013. Situación actual de los frutales caducifolios en el mundo y en Colombia. En: Miranda, D.; Fischer, G.; Carranza, C. (eds). Los frutales caducifolios en Colombia: Situación actual, sistemas de cultivo y plan de desarrollo. Soc. Col. Cienc. Hort. p.9-20.
51. PUENTES, G.; RODRÍGUEZ, L.F.; BERMÚDEZ, L. 2008. Análisis de grupo de las empresas productoras de frutales caducifolios del departamento de Boyacá. Agr. Col. 26(1):146-154.

52. PUPPO, L.; GARCÍA, M. 2010. Determinación del consumo de agua del duraznero por lisimetría. Rev. Bras. Eng. Agríc. Ambiental. 14(1):25-31.

53. RAMÍREZ, F.; KALLARACKAL, J. 2014. Ecophysiology of temperate fruit trees in the tropics. En: Daniels, J.A. (ed). Advances in environmental research. Vol. 31. Nova Science Pub. (Hauppauge, N. Y.). p.1-13.

54. RASEIRA, C.; NAKASU, B. 1989. Novas cultivares de nectarineira para o Sul do Brasil. Rev. Hortisul. 1:2223.

55. REIGHARD, G.L.; PARKER, M.L.; KREWER, G.W.; BRECKMAN, T.G.; BRUCE, W.W.; SMITH, J.; WHIDDON, J. 2001. Impact of Hurricanes on Peach and Pecan Orchards in the Southeastern United States. Hort Science. 36(2):250-252.

56. REAL, J.I. 1987. Estimating chill units at low latitudes. HortScience. 22(6):1227-1231.

57. RICHARDSON, E.A.; SEELEY, S.D.; WALKER, D.R. 1974. A model for estimating the completion of rest for 'Redhaven' and 'Elberta' peaches. HortScience. 9:331-332.

58. ROHDE, A.; BHALERAO, R. 2007. Plant dormancy in the perennial context. Trends Plant Science 12(5):217223.

59. ROM, C.R. 2003. Dormancy and acclimation. En: Baugher, T.A., Singha S. (eds). Concise encyclopedia of temperatue tree fruit. Food Product Press, New York. p.57-63.

60. ROMO, R.; DÍAZ, D.H. 1985. Root system and nutritional status of peaches under drip or flood irrigation in warm climates. Acta Hort. 173:167-175.

61. RUSSEL, D.M.; TOPP, B.L. 2002. Performance of Brazilian stone fruit varieties in a temperate region of Australia. Acta Hort. 592:149-155.

62. SHERMAN, W.P.; LYRENE, P.M. 1984. Biannual peaches in the tropics. Fruit Var. J. 38:37-39. 
63. TROMP, J. 1976. Flower bud formation and shoot growth in apple as affected by temperature. Scientia Hort. 5:331-338.

64. UNITED STATE DEPARTMENT OF AGRICULTURE USDA-. 2013. Natural Resources Conservation Service, Plant Database. Disponible desde internet en: http://plants.usda.gov/java/ClassificationServlet. (con acceso 15/12/13).
65. WESTWOOD, M.N. 1993. Temperate-zone pomology. Timber Press, Portland, ME. 523p.

Recibido: Junio 8 de 2014

Aceptado: Octubre 10 de 2014

\section{Como citar:}

Pinzón, E.H.; Cruz Morillo, A.; Fischer, G. 2014. Aspectos fisiológicos del duraznero (Prunus persica [L.] Batsch) en el trópico alto. Una revisión. Rev. U.D.C.A Act. \& Div. Cient. 17(2): 401-411. 